\title{
LA RÉCEPTION LITTÉRAIRE TRANSNATIONALE
}

\section{TRANSNATIONAL LITERARY RECEPTION}

Joseph Jurt*

\section{Introduction}

Le fait littéraire est constitué par trois éléments: le texte, sa production et sa réception. La critique littéraire a cependant à tour de rôle priviligié un de ces trois éléments.

\section{Le textualisme}

En Allemagne, par exemple, a prévalu après 1945 une approche immanente de la littérature, qui s'en tenait aux seules structures des textes et notamment aux procédés narratifs. ${ }^{1}$ La théorie des formes littéraires, développée en France notamment par Roland Barthes et Gérard Genette, s'inspirant de l'approche structuraliste, s'en tenait également au seul texte et à sa dimension synchronique. Barthes défendait à son tour la thèse de l'imperméabilité entre l'histoire et les œuvres. Paul Dirkx (2000; 2004) a parlé à juste titre de 'textualisme'. Si la poétique structurale s'était intéressée exclusivement aux systèmes et fonctions des formes s'en tenant à la stricte clôture du texte, on commençait pourtant dès le début des années soixante-dix à introduire ce que Louis Hay appela la troisième dimension du texte, c'est-à-dire la relation qu'entretiennent les œuvres modernes avec leur propre temporalité d'apparition en étudiant le processus générateur à travers une analyse de manuscrits.

\footnotetext{
* Professeur au Frankreich-Zentrum der l'université Albert Ludwig de Fribourg en Allemagne (Freiburg/ DE). E-mail: joseph.jurt@romanistik.uni-freiburg.de

1. Voir Jurt (1989).
} 


\section{Génétique textuelle}

Ainsi s'est constituée la critique génétique. Après l'obsession synchronique de la forme, on commença à s'intéresser au déroulement diachronique. Cette évolution s'était déjà annoncé dans les derniers écrits de Roland Barthes, par exemple dans son article sur "théorie du texte", paru en 1985 dans L'Encyclopedia universalis, où il admettait aussi l'historicité génétique au texte. Cette nouvelle démarche a été initiée en 1977 par le livre de Jean Bellemin-Noël Le texte et l'avant-texte qui, à travers une analyse des esquisses d'un poème de Milosz, plaidait une "poétique de brouillons". Tout en maintenant une théorie de structurale du texte, il se concentra sur la textualité spatio-temporelle sans recourir pourtant à un sujet producteur ou récepteur. La critique génétique s'est constituée dans la suite comme un paradigme important des recherches littéraires en France.

La génétique textuelle, en revendiquant la théorisation d'une dimension historique, à l'intérieur même de l'écrit, remarque à juste titre Pierre Marc de Biasi, introduit donc "ce qui faisait le plus cruellement défaut aux analyses formelles: l'étendue inexploré d'un nouvel objet structuré par le temps" (BIASI, 1985, p. 468). La critique génétique cherchera donc à défınir selon Jacques Neefs "dans les états successifs ou concurrents d'un ensemble d'écrits, les relations significatives d'une activité crátrice, et à élaborer une "poétique de l'écriture" (1990, p. 22).

La critique génétique s'efforce de saisir la genèse des textes à travers une transcription et un examen minutieux de tous les états des manuscrits des écrivains auxquels on attribue un statut esthétique spécifique et non seulement celui d'un simple 'matériau'. La critique génétique est devenu un paradigme critique très important. $^{2}$ Jaques Neefs a cependant radicalement distingué la critique génétique qui s'attache à la textualité en mouvement d'une œuvre par l'étude de ses avant-textes, des broullions, de ses versions diverses de la notion du 'génétique' telle qu'elle apparaît depuis Lucien Goldmann, dans les méthodes sociologiques du "structuralisme génétique” (NEEF, 1990, p. 23).

\section{Génétique sociale}

On reconnaîtra que ce génétisme, qui établit des rapports d'homologie entre la structure des contenus des oeuvres et la vision du monde élaborée par la conscience collective d'un groupe social, déterminé lui-même par la situation sociale, politique, économique donnée, opère d'une manière assez mécanique et réductrice. Le caractère réducteur de ce modèle génétique ne peut pourtant pas être une raison pour évacuer totalement toute explication de type social.

Il y a des modèles d'explication sociale plus fins que celui du structuralisme génétique goldmannien qui tiennent compte notamment d'un fait extrêmement important: l'autonomisation de la production culturelle au cours du XIX ${ }^{\mathrm{e}}$ siècle. Je pense, évidemment, à la théorie du champ littéraire. Pierre Bourdieu estime ainsi que l'analyse des versions successives d'un texte "revêtirait sa pleine force explicative si elle visait à reconstruire [...] la logique du travail d'écriture entendu comme recherche 
accomplie sous la contrainte structurale du champ et de l'espace des possibles qu'il propose" (BOURDIEU, 1992a, p. 277). On comprendrait, selon lui, mieux:

les hésitations, les repentirs, les retours si l'on savait que l'écriture, navigation périlleuse dans un univers de menaces et de dangers, est aussi guidée, dans sa dimension négative, par une connaissance anticipée de la réception probable, inscrite à l'état de potentialité dans le champ; que [...] l'écrivain tel que le conçoit Flaubert est celui qui s'aventure hors des routes balisées de l'usage ordinaire et qui est expert dans l'art de trouver le passage entre les périls que sont les lieux communs, les 'idées reçues', les formes convenues. (BOURDIEU, 1992a, p. 277-278)

La génétique textuelle et la génétique sociale ne me semblent donc pas comporter de rapports d'exclusion, mais de complémentarité3.

Cette complémentarité entre génétique textuelle et génétique culturelle a ainsi été démontrée par Henri Mittérand relevant, à propos des textes de Zola, que dans les premières lignes d'une ébauche écrites dans une relative spontanéité se révélait le contact le plus direct avec le discours social, que Zola partait des conceptions collectives de la doxa contemporaine et qu'à travers le processus de l'écriture, les oeuvres se transformaient d'objets en sujets. A travers la dynamique de l'écriture, cette interaction entre social et esthétique serait donc parfaitement saisissable (MITTERAND, 1989).

Ce qui frappe de toute évidence c'est la mise en valeur, de part et d'autre, de la dimension génétique. Pierre Bourdieu, en appelle à ce qu'on travaille "de manière concertée [...] pour s'atteler à une vraie théorie de la production littéraire" (BOURDIEU, 1992b, p. 111). Le terme de genèse apparaît déjà dans le sous-titre de son ouvrage. La finalité de l'analyse scientifique c'est, à ses yeux, "de porter au jour ce qui rend l'oeuvre d'art nécessaire, c'est-à-dire la formule informatrice, le principe générateur [...]" (BOURDIEU, 1992a, p. 14). À travers le personnage de Frédéric de L'Education sentimentale et la description de sa position dans l'espace social, Flaubert livre, d'après Pierre Bourdieu, "la formule génératrice qui est au principe de sa propre création romanesque" (BOURDIEU, 1992a, p. 55).

Seule une analyse de la genèse du champ littéraire dans lequel s'est constitué le projet flaubertien peut conduire à une compréhension véritable et de la formule génératrice qui est au principe de l'oeuvre et du travail grâce auquel Flaubert est parvenu à la mettre en oeuvre, objectivant, dans le même mouvement, cette structure génératrice et la structure sociale dont elle est le produit. (BOURDIEU, 1992a, p. 76)

La dimension sociale qui peut expliquer la genèse d'une œuvre littéraire ou picturale n'est pas pour Bourdieu la société globale, mais le champ littéraire ou artistique qui est le produit d'un processus historique et qui se défınit par une autonomie (relative) par rapport aux champs politiques ou économiques. C'est un "univers social qui est le monde des peintres, des critiques, des artistes, etc., qui obéit à des lois sociales, à des lois de fonctionnement, à l'intérieur duquel le peintre est lui-même inséré et à 
l'intérieur duquel il travaille", écrit Bourdieu au sujet de Manet. Lorsqu'il [Manet] peint, par exemple, il n'est pas tout seul en face de son tableau: "il a dans la tête d'autres peintres du passé et du présent, et aussi un public, celui auquel s'adresse son tableau" (BOURDIEU, 2013, p. 403).

Ce n'est donc pas le champ seul qui conditionne l'acte de la production de l'œuvre. Il y a une interaction entre l'auteur doté d'un capital (culturel, social et économique) spécifique et de dispositions qui lui sont propres: "Les dispositions sont cet ensemble de manières d'être permanentes que chacun de nous a acquises de son univers social, c'est-à-dire schèmes de perception, d'appréciation et d'action qui sont des principes générateurs unitaires et systématiques de pratiques, d'œuvres, d'expression en général" (BOURDIEU, 2013, p. 451).

Quand on compare champ littéraire et champ artistique, on peut relever un certain nombre d'invariants qui transcendent les deux champs. ${ }^{4}$ Parmi ces invariants il faut compter le caractère des produits culturels défınis par Pierre Bourdieu comme 'biens symboliques' se distinguant par leur double nature (signification et marchandise). La valeur symbolique des produits culturels n'existe pas en tant que telle; les objets matériels créés par l'artiste ou l'écrivain ne deviennent œuvres d'art que lorsqu'ils sont reconnus en tant que tels - par des instances de sélection et de consécration compétentes. Une sociologie de l'art et de la littérature ne peut donc se contenter d'envisager les producteurs directs des œuvres dans leur matérialité comme les seuls constituants de l'œuvre; elle doit tenir compte également de ceux qui créent le sens et la valeur sociale de l'œuvre: les critiques, les éditeurs, les directeurs de galeries, les membres des instances de consécration, les Académies, les salons, les jurys et l'ensemble des personnes qui contribuent à former des 'consommateurs' capables de connaître et de reconnaître l'œuvre d'art en tant que telle, à savoir comme valeur (BOURDIEU, 1980; 1984).

\section{La réception}

L'extrême valorisation de la créativité explique que l'intérêt de la théorie de la littérature se soit porté par préférence sur la genèse textuelle et sociale des œuvres et beaucoup moins sur la réception, sur la lecture ${ }^{5}$. Or, le généticien est d'abord un lecteur, comme l'a souligné Almuth Grésillon (1993). Il doit lire et déchiffrer les manuscrits afin d'exprimer des hypothèses sur la genèse. La lecture renvoie ainsi de nouveau à la production; on ne saurait séparer nettement les deux dimensions. Les auteurs se sont souvent examinés comme des lecteurs. Les auteurs sont aussi des lecteurs réels; ils consultent parfois des bibliothèques entières avant d'écrire, notamment les oeuvres se distinguant par un certain degré de référentialité.

Qu'on pense aux 'Dossiers d'enquête' de Zola ou de Flaubert. Foucault soulignait dans son étude sur La Tentation de SaintAntoine que chaque auteur écrit à partir d'une bibliothèque. Cervantes écrit à partir d'une bibliothèque, à partir des romans de chevalerie, des romans picaresques, des romans byzantins. En même temps il thématise la lecture, les effets de la lecture

4. Voir à ce sujet Bourdieu (1980).

5. Sur l'aspect de la réception voir Jurt (1979; 1980). 
comme plus tard Flaubert. On a parlé d'Emma Bovary comme d'un don Quijote féminin. Les deux ratent leur vie à cause d'un mauvais usage de la lecture.

L'écrivain tout en écrivant, "se relit"; Almuth Grésillon a cité un texte de Sartre qui distingue la relecture de l'auteur de la lecture première du lecteur:

Or, l'opération d'écrire comporte une quasi-lecture implicite qui rend la vraie lecture impossible. Quand les mots se forment sous sa plume, l'auteur les voit, sans doute, mais il ne les voit pas comme le lecteur puisqu'il les connait avant de les écrire; son regard n'a pas pour fonction de réveiller en les frôlant des mots endormis qui attendent d'être lus, mais de contrôler le tracé des signes, c'est une mission purement régulatrice, en somme, et la vue ici n'apprend rien, sauf de petites erreurs de la main. (SARTRE, 1948, p. 52-53)

Paul Ricoeur a mis en relief la double dimension du texte littéraire - ensemble consistant et structuré du point de vue de l'auteur et en même temps, ouvert aux interprétations des lecteurs - qui suscite deux points de vue de la critique littéraire, celui de la genèse et celui de la réception. La confrontation de ces deux dimensions se révèle inévitable, le sens partant $d u$ texte mais s'achevant dans la lecture: "Une dialectique infınie est déclenchée entre la structuration interne du texte et toutes les activités de déstructuration - restructuration relevant de la lecture. Cette dialectique fait de l'oeuvre l'effet commun de l'auteur et du lecteur" (RICOEUR, 1989, p. 226).

C'est Jean-Paul Sartre qui a été un des premiers à concevoir la lecture comme élément constitutif de la constitution $\mathrm{du}$ sens d'une œuvre. Dans son essai fondamental Qu'est-ce que la littérature?, il défınit l'ouvrage de l'esprit par le public auquel il s'adresse, se refusant à considérer la condition de l'auteur comme facteur déterminant: "Le milieu est une vis à tergo; le public au contraire est une attente, un vide à combler, une aspiration, au figuré et au propre [...] Et je suis si loin de repousser l'explication de l'œuvre par la situation de l'homme quej'ai toujours considéré le projet d'écriture comme le libre dépassement d'une certaine situation humaine et totale" (SARTRE, 1970, p.96). L'œuvre écrite en vue d'un public n'existe encore que sur le mode du possible. Ce n'est que la lecture qui peut conférer la vie aux signes abstraits de l'ouvrage. L'effet calculé par l'artiste ne s'actualise que lors de la perception par le lecteur. La notion traditionnelle de lecture s'est cependant révélée inapte à élucider ce processus; la lecture n'est pas seulement perception passive d'un objet donné une fois pour toutes et que nous pourrions regarder sans y mettre rien de nous-mêmes.

La lecture est un processus actif, ce qui a été pertinemment exposé par Sartre: "Le lecteur invente tout dans un perpétuel dépassement de la chose écrite" (SARTRE, 1970, p. 57). L'auteur de Qu'est-ce que la littérature? propose pour cet acte de lecture, constituant le mode d'existence de l'œuvre d'art, la formule heureuse de “création dirigée" (SARTRE, 1970, p. 57): "D'une part, en effet, l'objet littéraire n'a d'autre substance que la subjectivité du lecteur [...] Mais d'autre part, les mots sont là comme des pièges pour susciter nos sentiments et les réfléchir vers nous [...] Ainsi, pour le lecteur, tout est à faire et tout est déjà fait; l'œuvre n'existe qu'au niveau exact de ses capacités" (SARTRE, 1970, p. 57-58). L'œuvre n'existe - telle est la thèse centrale de Sartre - qu'en tant que lue. Le 
lecture - "synthèse de la perception et de la création” - requiert le texte écrit. Écriture et lecture sont ainsi les deux faces d'un seul processus.

\section{L'esthétique de la réception}

L'apport le plus spécifiquement allemand à la critique littéraire des dernières décennies semble être l'esthétique de la réception développée par l'Ecole de Constance dont les pères fondateurs ont été le romaniste Hans Robert Jauss et l'angliciste Wolfgang Iser. C'est la "leçon inaugurale" de Jauss, prononcée à Constance en 1967, qui a tenu lieu de manifeste de cette nouvelle école (Literaturgeschichte als Provokation der Literaturwissenschaft) ${ }^{6}$. Cette "leçon" a été sans doute également inspirée par le contexte, par l'élan nouveau qui soufflait dans cette nouvelle université au bord du lac de Constance, destinée à l'époque à devenir un Harvard allemand. Par le titre de sa leçon inaugurale (l'histoire littéraire comme défi), Jauss signalait qu'il entendait restaurer l'historicité de la littérature, bannie par l'analyse immanente. En même temps, il s'agissait pour lui de s'opposer à l'objectivisme historique. Dans sa Leçon, Jauss a déclaré vouloir remplacer l'esthétique de la production traditionnelle par une esthétique de la réception et de l'effet. L'historicité du fait littéraire ne résiderait pas dans la production, mais dans la réception. Le fait historique, c'est, pour lui, la lecture, l'actualisation du texte par le lecteur. L'histoire littéraire, selon cette optique, c'est l'histoire des actualisations successives du texte par le lecteur:
"L'histoire de la littérature est un processus de réception et de production esthétique qui s'accomplit dans l'actualisation des textes littéraires par le lecteur qui lit, le critique qui réfléchit et l'ecrivain qui produit de nouveau" (JAUSS, 1970).

Afın de ne pas réduire l'histoire de la réception à un inventaire de réactions individuelles et purement psychologiques, Jauss introduit le concept "horizon d'attente”, emprunté à la sociologie de Mannheim et conçu comme un système de référence trans-individuel. Le moment de la lecture est décrit comme la fusion de l'horizon intratextuel et de l'horizon d'attente (extratextuel) de l'époque. Ce concept de fusion des horizons est emprunté à Gadamer (qui conçoit la lecture comme un dialogue entre l'interprète et le texte, une intégration du passé dans le présent, le lecteur étant influencé par la tradition et influençant à son tour celle-ci). L'histoire de la réception se rattache donc à l'histoire de l'effet (Wirkungsgeschichte) proposée par Gadamer, tout en se présentant comme un changement radical de perspective dans l'approche littéraire (esthétique de la réception $v s$ esthétique de la production). La proposition de Jauss s'insère dans la tradition de la tendance philosophique dominante en Allemagne: l'hermeneutique; celle-ci souligne l'importance du sujet et de la situation historique lors du processus de perception (contre l'objectivisme) et érige la tradition (des interprétations antérieures) en catégorie trans-subjective (contre le subjectivisme).

Jauss présente la "distance esthétique" comme un critère d'évaluation esthétique

6. Frankfurt, Suhrkamp, 1970. Traduction française: Pour une esthétique de la réception, Paris, Gallimard, 1978. 
des textes: à savoir l'écart entre l'œuvre et l'horizon d'attente constitué par la tradition d'un genre, la topique, les métaphores. L'écart ou l'innovation sert de paramètre mesurant la valeur esthétique. Ce paramètre est emprunté aux formalistes russes; on accorde ainsi à une catégorie historique qui est tout au plus pertinente pour la littérature moderne une pertinence universelle. D'autre part, on attribue à l'horizon d'attente intralittéraire (les signaux du texte) la priorité face à l'horizon extra-littéraire. En situant les normes dans l'œuvre, Jauss retombe dans une ontologisation de l'œuvre qu'il s'était proposer de conjurer.

La société est présente, pour Jauss, à l'extérieur du fait littéraire en tant que cadre. Les répercussions sont, selon lui, une conséquence de la communication littéraire. Par l'acte de réception, écrit Jauss, l'expérience littéraire peut entrer dans la vie pratique (Lebenspraxis) du lecteur, préformer sa vision du monde et influer ensuite sur son comportement. Jauss méconnaît que le fait littéraire est lui-même un fait social. Pour lui, le contact avec l'œuvre peut contribuer à élargir l'expérience propre du lecteur grâce à l'expérience d'autrui, mais cette expérience ne semble pas avoir d'incidence sur l'action pratique (JAUSS, 1984, p. 822). Claude Piché remarque à ce sujet avec raison: "Pour le moment, nous ne pouvons concevoir l'application 'esthétique' autrement que comme cette expérience qui contribue pour le lecteur à renforcer ou à déplacer les canons reconnus de l'esthétique, sans plus" (PICHÉ, 1984, p. 187).

On ne niera pourtant pas que l'introduction des termes d'horizon d'attente et de distance esthétique a été féconde. Or, les propositions de Jauss en restent le plus souvent au niveau de la théorie; ce sont des thèses (ou plutôt des hypothèses) qui doivent être vérifiées à la lumière d'enquêtes empiriques; celles-ci devraient s'inscrire dans deux directions: d'une part, il s'agit de fonder la reconstitution de l'horizon d'attente d'un moment donné sur une base documentaire représentative, voire exhaustive; d'autre part, il importerait de saisir les conditionnements extralittéraires $\mathrm{du}$ processus de la réception.

Ce sont ces deux intérêts qui nous ont incité à nous consacrer à un projet de recherche qui devait élucider de plus près le processus de la réception. Ce processus nous paraît saisissable pour une période historique dans les traces écrites que nous a laissées la critique littéraire ${ }^{7}$. Pour cette raison, nous avons établi dans un premier temps un inventaire qui se voulait exhaustif de toutes les réactions provoquées par l'œuvre littéraire d'un auteur de l'entredeux-guerre, en l'occurrence celle de Georges Bernanos; nous avons également pris en considération un certain nombre de réactions suscitées par les romans de Gide et de Malraux. Tous ces comptes rendus ont été analysés à l'aide d'une grille élaborée à ce dessein. Dans une synthèse, nous avons ensuite cherché à établir une classification significative des réactions ainsi qu'un relevé des critères qui informent le jugement littéraire. On a classé ainsi les réactions, d'après les orientations politiques de la presse, en huit courants idéologiques (extrême droite, droite, bourgeoisie, catholiques, modérés, centre littéraire, gauche radical, gauche socialiste

7. Au sujet de la critique littéraire journalistique, voir Jurt (1980). 
et gauche communiste). Notre analyse a pu mettre en lumière une assez grande cohérence des réactions à l'intérieur des courants donnés; nous avon pu constater que les jugements littéraires sont fortement influencés par les présupposés idéologiques respectifs. En examinant la forme des critiques, on a pu déceler deux types de réactions: d'une part une critique judicative qui opère à partir d'un modèle, d'un idéal (littéraire, référentiel, socio-culturel, moral, doctrinal); l'œuvre est alors jugée sa conformité à cet idéal; d'autre part, nous avons relevé une critique compréhensive - pratiquée par une minorité parmi les interprètes - qui entend moins juger les œuvres que les comprendre; cette critique n'isole pas certains aspects particuliers de l'œuvre, elle cherche au contraire à intégrer un maximum d'éléments du texte dans son interprétation.

A la lumière des résultats de notre analyse, il a été possible de vérifier les principales hypothèses de l'esthétique de la réception; celle-ci postule, nous l'avons vu, un horizon d'attente constitué presque exclusivement par des expériences et des connaissances littéraires. Cette hypothèse n'a pas été confirmé par notre analyse. Les jugements des interprètes ne sont pas déterminés en premier lieu par des critères esthétiques; les critères d'appréciation sont le plus souvent d'ordre extra-littéraire; les critères esthétiques servent maintes fois à corroborer un préjugement idéologique préalable.

D'après une seconde hypothèse de H. R. Jauss, la critique littéraire tend à fixer les normes (esthétiques) d'une époque et manifeste alors sa déception lorsqu'une œuvre innove et transgresse l'horizon d'attente donné. Or, dans notre analyse, nous avons recensé toute une série de réactions qui valorisent les traits innovateurs et originaux des œuvres littéraires. La majorité des jugements critiques sont cependant émis à partir d'un horizon d'attente conservateur, s'inspirant des modèles mentionnés ci-dessus. $\mathrm{Vu}$ ces valorisations divergentes de l'innovation, on ne saurait donc maintenir le postulat d'un horizon d'attente uniforme pour toute une époque.

L'esthétique de la réception ne saurait remplacer, comme H. R. Jauss l'avait formulé dans ses premiers écrits, une esthétique de la production; elle complète plutôt celle-ci en mettant en valeur la présence du lecteur dans le texte et l'importance du lecteur réel pour la concrétisation. Mais on n'oubliera pas pour autant que le processus dynamique qui englobe à la fois la production du texte à partir de ses conditions de possibilité spécifique et la concrétisation du sens à partir des appels inhérents au texte; la concrétisation est à son tour en tant que production du sens un processus actif qui peut provoquer de nouvelles productions de textes.

\section{La réception littéraire transnationale}

La réception littéraire a été analysée de préférence à l'intérieur d'un espace national homogène. Si l'on allait au-delà, c'est surtout pour étudier les relations au 'sommet' entre Goethe et Racine ou Verga et Zola. Ceci s'explique aussi par le fait que les études littéraires sont marquées jusqu'à nos jours par le mythe du 'grand écrivain', mythe que les intéressés n'ont aucun intérêt à détruire. Anne Boschetti a ainsi rappelé que l'auteur d'une oeuvre 'originale' jouissait d'un prestige supérieur à celui de toutes les autres catégories (éditeurs, commentateurs, professeurs, traducteurs) 
en raison de la croyance dans le 'génie créateur', comme don individuel qui serait la cause première et le principe explicatif de l'oeuvre. Les autres agents culturels seraient par ailleurs souvent les premiers à reconnaître implicitement la supériorité de la 'création' en voyant dans leur métier un moyen pour être créateurs par procuration (1994, p. 51-52).

Quand on analyse la réception d'oeuvres littéraires, on ne saurait s'en tenir à la seule catégorie du 'grand auteur' ou de la 'grande oeuvre'. Il faudra, en effet, envisager l'ensemble des agents impliqués dans ce processus. On a pu ainsi reprocher, non sans raison, à 'l'esthétique de la réception', qui entendait en premier lieu évaluer la qualité esthétique des oeuvres à travers la différence esthétique entre le produit créé et l'horizon d'attente, de négliger les conditions sociales $\mathrm{du}$ processus de la réception. On ne rend raison de ce processus que si l'on n'isole tel ou tel agent, mais seulement en considérant ceux-ci comme faisant partie d'un ensemble ou d'un système et que je désignerais, avec Pierre Bourdieu, comme un champ.

S'il faut tenir compte de tous les agents impliqués dans le processus de la réception, il ne faudra surtout pas négliger les facteurs spécifiques qui entrent en ligne de compte lorsqu'une oeuvre est reçue dans un champ étranger. C'est encore Yves Chevrel qui a, dans un des premiers numéros des Cahiers d'Histoire de Littératures Romanes, pertinemment relevé les traits spécifiques caractérisant la réception critique des oeuvres étrangères. Ce qui distingue l'oeuvre étrangère, c'est selon Chevrel la grande distance qui la sépare du champ d'accueil; le contexte extra-littéraire y entre en ligne de compte, par exemple le statut des relations officielles avec la nation d'origine. La littérature étrangère est toujours soumise à une explication, suivant souvent une démarche ramenant l'inconnu (l'étranger) au connu (la tradition française universalisante). Le procédé le plus usuel est la simple mise en parallèle; on invite ensuite les lecteurs à découvrir la trace de leur propre littérature ou bien on affirme que tout a été déjà dit par la littérature française. Yves Chevrel relate à ce sujet des propos formulés par Zola en 1897 dans La Revue blanche: "De même que notre vin de Bordeaux, dit-on, gagne à faire le voyage des Indes, il est certain que quelques-unes de nos idées, en passant par le génie du Nord, ont pris une ampleur et une intensité admirables" (1977, p. 338-343). C'est Pierre Bourdieu qui a très bien défıni les conditions spécifiques de la réception d'oeuvres scientifiques et littéraires étrangères. Il constate que les textes circulent souvent sans leur contexte. Les récepteurs, insérés dans un champ de production différent, les réinterprètent en fonction de la structure du champ de réception. Le sens et la fonction d'une oeuvre étrangère seraient ainsi déterminés au moins autant par le champ d'accueil que par le champ d'origine. La fonction dans le champ d'origine serait d'une part souvent ignorée, le transfert d'un espace à l'autre se ferait d'autre part à travers toute une série d'opérations sociales, une opération de sélection, une opération de marquage et une opération de lecture, les lecteurs appliquant à l'oeuvre souvent des catégories de perception et des problématiques de leur propre champ.

Les effets structuraux qui à la faveur de l'ignorance, rendent possibles toutes les transformations et les déformations créées à des usages stratégiques des textes et des auteurs, peuvent s'exercer en dehors de toute inter- 
vention manipulatrice. Les différences sont si grandes entre les traditions historiques [...] que l'application à un produit culturel étranger des catégories de perception et d'appréciation acquise à travers l'expérience d'un champ national peut créer des oppositions fictives entre des choses semblables et de fausses ressemblances entre des choses différentes. (BOURDIEU, 1990)

Si la référence à la nation et à la culture nationale d'accueil joue un grand rôle lors de la réception, il y a eu pourtant des variations historiques. C'est surtout depuis le romantisme que ce recours a gagné en importance. La littérature des Lumières s'est défınie par son universalisme, encore que cet universalisme ait été l'expression d'une mince couche de la population. On peut même affirmer que le cosmopolitisme du siècle des Lumières n'était universel qu'en apparence; car il ne s'agissait pas plus d'un échange d'idées et d'oeuvres entre partenaires culturels égaux que de la suprématie du modèle culturel français qui a pu fonctionner grâce au stratagème qui consistait à déclarer les valeurs de la civilisation française comme universelles. Cette suprématie a pu fonctionner aussi $\mathrm{du}$ fait que des membres d'une élite nonfrançaise avaient internalisé le modèle tout en l'instrumentalisant comme signe de distinction face à des classes inférieures (ESPAGNE e WERNER, 1987) ${ }^{7}$.

C'est contre cette civilisation aristocratique d'inspiration française et apparemment transnationale, jugée superficielle et aliénatrice, que les intellectuels allemands d'origine bourgeoise opposaient leur conception de la Kultur. C'est notamment Justus Möser qui devait, en réponse à Frédéric II, plaider en 1781 pour une identité culturelle allemande à travers son écrit Über die deutsche Sprache und Literatur, dénonçantl'idéal littéraire français comme exclusivement aristocratique et louant Goethe et les auteurs anglais d'avoir intégré dans leurs écrits la dimension du peuple. A ses yeux, il ne saurait y avoir de normes poétiques universelles et il avait recours à une conception organiciste de la culture. Il y aurait une culture spécifique pour chaque peuple, la variété et non pas l'uniformité étant la loi de l'univers. Cette conception organiciste de la culture prévaudra notamment chez Herder pour qui l'individualité de chaque peuple se traduit par sa langue et qui cherche dans les textes originaux la manière de pensée, le principe originaire de la nature, son 'Volksgeist'. La conception organiciste de la culture, qu'on pouvait trouver chez Herder et Möser, aurait dû aboutir à une reconnaissance de légitimité des autres cultures. La littérature nationale allemande s'est cependant affırmée, dès la fin du XVIII ${ }^{\mathrm{e}}$ siècle, notamment contre la prédominance française. Herder ne voyait dans la tragédie française classique que du mensonge et du galimatias, une marionnette dépourvue d'esprit, de vie et de vérité. Au cours du $\mathrm{XIX}^{\mathrm{e}}$ siècle, on ne cessera de critiquer

7. "La culture française des XVIII ${ }^{e}$ et $\mathrm{XIX}^{\mathrm{e}}$ siècles passe généralement pour avoir été peu ouverte sur le monde extérieur. Historiquement, cet égocentrisme est fondé sur une position dominante à l'intérieur de l'Europe. Forte de son rayonnement auprès des élites européennes, la culture nationale se suffisait pour ainsi dire à elle-même. La Révolution a repris en la politisant cette attitude qui, il faut le souligner, est largement antérieure à l'éveil des nationalismes et se confond pour une large part avec l'universalisme des Lumières” (1987, p. 971). 
l'orientation de la littérature allemande antérieure suivant le modèle étranger, notamment les littératures romanes.

Le nationalisme littéraire exclusif n'était pourtant pas la seule réaction destinée à dépasser le cosmopolitisme aristocratique. Un autre modèle se manifestait à travers le concept de la Weltliteratur de Goethe stipulant l'échange entre les littératures ${ }^{8}$ ainsi que dans les écrits de Mme de Staël. Mme de Staël partait aussi d'une conception culturelle organiciste. L'idée de la légitimité d'une littérature nationale impliquait cependant chez elle la reconnaissance de la légitimité identique des littératures des autres nations. Il importait de reconnaître ce qui est étranger dans son originalité en la rapportant aux conditions historiques spécifiques, ce que Mme de Staël tentait de faire dans son ouvrage De la littérature considérée dans ses rapports avec les institutions sociales (1800). A partir d'une telle reconnaissance mutuelle, un échange entre les cultures est possible. Mme de Staël a ainsi, selon Pierre Macherey, dépassé le particularisme romantique qui affirme l'autonomie radicale de chaque culture et l'universalisme classique qui confond toutes les cultures à l'intérieur d'un modèle idéal abstrait. La thèse qu'elle avait formulée dès la fin du XVIII siècle était tout à fait novatrice: "Il n'y a d'identité culturelle qu'à l'intérieur du rapport culturel qui rassemble toutes les cultures en les opposant entre elles" (1988, p. 425).
L'autonomie littéraire se manifestait également dans le domaine des études universitaires. La littérature, qui, certes, s'était inspirée des oeuvres de l'Antiquité et qui avait intégré au cours du XVIII ${ }^{\mathrm{e}}$ siècle l'empirisme anglais, passait pour universelle, mais cet universalisme était en fin de compte très gallocentrique et peu ouvert aux autres cultures. Dans son livre Le paradigme de l'étranger, Michel Espagne a démontré que ce n'est qu'en 1830 qu'on a créé à Paris la première chaire de littérature étrangère constituant ainsi par opposition une 'littérature français' dénuée de sa prétention universaliste. L'étranger pour la France du $\mathrm{XIX}^{\mathrm{e}}$ siècle était la culture allemande; et les instruments intellectuels à travers lesquels on cherchait à comprendre les cultures étrangères étaient empruntés à la tradition allemande: philologie, historisme, théorie de l'esprit national. Il ne s'agissait pourtant guère d'une vision désintéressée de la culture étrangère; celleci a été plutôt instrumentalisée au profit des besoins nationaux ${ }^{9}$. Le concept de la littérature étrangère impliquait, comme le remarque Michel Espagne, le principe de la différenciation; depuis 1879, on instruira une chaire pour la littérature de l'Europe du Sud et en 1901 on créa une chaire consacrée à la langue allemande et anglaise.

La référence nationale a déterminé la réception de la littérature de part et d'autre encore au XX $\mathrm{XX}^{\mathrm{e}}$ siècle. Doris Harrer constate

\section{Voir Jurt (2012).}

9. "Les professeurs ayant en charge cette discipline ont au contraire souvent le sentiment de mettre en évidence une diffusion de la France à travers l'Europe, le choix des auteurs étrangers qu'ils prennent en considération reste très limité et commandé par des considérations purement nationales, par des besoins spécifiques de la culture d'accueil [...] Ce qui s'opère en revanche, c'est une instrumentalisation variée et complexe des cultures voisines, une instrumentalisation peut-être inconsciente, mais dont les différentes figures sont assez marquées pour mériter d'être successivement éclairées” (ESPAGNE, 1993, p. 15-16). 
ceci dans la conclusion de sa thèse sur la réception de la littérature française pendant la République de Weimar. La littérature française était devenue, selon cet auteur, dans l'Allemagne de la République de Weimar, marquée par une crise d'identité nationale, un moyen de la recherche de soi; les uns entendaient maintenir une entité allemande s'opposant à l'étranger incarnée par la littérature française; des critiques proches de la gauche entendaient en revanche dépasser 'l'essence' allemande en s'adaptant à l'étranger représenté également par la littérature française. Ceux qui cherchaient à comprendre la littérature française en tant que telle comme Walter Benjamin se trouvaient plutôt en marge ou à distance des milieux dominants de la République de Weimar (1987, p. 182-183).

Ernst Robert Curtius a ainsi constaté dans un article publié en 1928 que chaque partenaire de ce dialogue francoallemand se servait inconsciemment des normes de sa propre culture comme critère d'appréciation de la culture de l'autre. Mais Curtius n'avait lui-même pas pu échapper à ce mode de perception. S'il avait voulu présenter dans son livre sur les précurseurs de la nouvelle France (Die Wegbereiter des neuen Frankreich [1919]) une image autre que celle d'une France décadente, il recourait tout de même à des stéréotypes convenus en associant la France à un état d'agrégation solide et l'Allemagne à un état liquide. Dans les auteurs tels que Gide, Romain Rolland, Claudel, Suarès, Péguy, il voyait les représentants d'une 'jeune' France correspondant à l'image que l'Allemagne se faisait d'elle même - représentant le devenir. Curtius croyait découvrir chez les auteurs un sentiment de valeurs qui aurait une "mesure commune" avec celui des Allemands; il mettait en relief, chez ces écrivains, les catégories de l'intensité de la vie et du vécu, nouvel élan que Bergson et Nietzsche semblaient confırmer à partir d'une philosophie vitale ${ }^{10}$.

Lors d'une enquête empirique sur les modalités socio-historiques de la lecture, Jacques Leenhardt et Pierre Józsa ont dû se rendre à l'évidence que l'idée selon laquelle chaque lecteur se trouve face aux chefsd'oeuvre de la littérature mondiale dans une situation de lecture identique était à réviser. "L'étude empirique de la lecture montre en effet que l'unité de la raison et l'unicité du texte jouent un rôle secondaire dans l'acte de lecture. Selon le lieu et le temps, la hiérarchisation des catégories de la perception et de l'évaluation varie de telle sorte qu'il s'avère nécessaire de considérer que l'objet visé dans l'acte de lire est la lecture elle-même plus encore que le texte" (LEENHARDT, 1988, p. 75-76).

Lors de leur enquête empirique, Leenhardt et Józsa ont fait lire un roman français (Les Choses de Pérec) et un roman hongrois (Le cimetière de rouille de Fejes) à un échantillon représentatif de lecteurs en France et en Hongrie. Les résultats étaient loin d'être identiques. En Hongrie a prévalu pour le roman français une lecture moraliste et pour le roman hongrois une lecture sociologique, politique, alors qu'en France a dominé pour les deux romans une lecture analytique et sociologique. Si la question politique évoquée dans les romans transite en Hongrie par l'identification, cela prouve, aux yeux des auteurs, que le discours universaliste n'y est pas à la disposition de chacun. En

10. Voir aussi Jurt (1995). 
revanche, la capacité dans laquelle se trouve théoriquement tout Français d'embrasser les problèmes dans leur pleine complexité peut paraître comme un effet de la prévalence en France du discours universaliste" (LEENHARDT, 1982).

\section{Conclusion}

L'analyse de la réception des oeuvres étrangères qui est celle de la circulation internationale des oeuvres et des idées n'est pas qu'un sujet académique. Dans le domaine des échanges de biens matériels, la mondialisation semble de plus en plus prévaloir; ceci n'est nullement le cas pour les échanges culturels où l'on semble assister à une évolution inverse. Les domaines linguistiques même à l'intérieur des Etats-Nations se différencient de plus en plus. L'Europe est divisée en cultures nationales étroitement cloisonnées et chacun des pays ignore trop ce qui se passe, tant dans le domaine du débat des idées qu'en matière de création littéraire chez ses voisins, surtout s'ils n'appartiennent pas à la même aire linguistique ${ }^{11}$.

Il importe donc d'analyser de près les facteurs qui déterminent le transfert (ou le non-transfert) d'oeuvres et d'idées d'un champ national à l'autre; ceci devrait permettre à éclaircir les causes de beaucoup de malentendus structurels qui caractérisent les relations intellectuelles trans-nationales et d'ouvrir la voie vers un dialogue rationnel. ${ }^{12}$

A partir des propositions de Pierre Bourdieu dans son article précité "Les conditions sociales de la circulation internationale des idées" s'est constitué un réseau de recherches financé par la communauté européenne sous la dénomination ESSE. Pour un espace européen des sciences sociales (2004-2008) qui s'est consacré à l'analyse des processus de réception transnationaux de la littérature et des idées.

Il s'agissait de saisir les mécanismes d'importation et d'exportation des oeuvres des vingt dernières années sur un plan international. L'angle de vue sociologique s'élargit ici, s'agissant de l'échange de biens matériels et symboliques, au droit et à l'économie. C'est notamment le contexte de la mondialisation (économique) et ses conséquences pour l'échange intellectuel qui ont été pris en considération. Gisèle Sapiro a dirigé dans ce contexte trois ouvrages collectifs qui portent sur la problématique de la réception par le biais des traductions. ${ }^{13}$ D'autres études élaborées au sein de ce réseau ont porté sur des processus de réception concrets: ainsi la réception de Kant (BOUREL, 2009),

11. Voir ainsi les propos de Roger-Paul Droit à ce sujet: "Nous conversons par satellite d'un continent à l'autre. Images télévisées, messages télécopiés, ordres informatisés transitent, instantanément, aux antipodes. Mais de longues années, des décennies parfois, sont nécessaires pour qu'une oeuvre importante passe le Rhin, franchisse Alpes ou Pyrénées, traverse la Manche ou la Méditerranée. Nous ignorons avec constance des penseurs importants de pays voisins, qui nous toucheraient de près, alors que nous sommes avertis du déraillement d'un train ou d'un cas de vache folle. Ce n'est pas le moindre paradoxe de ce siècle: les affaires, politiques ou financières, les faits futiles aussi, ignorent les distances et le temps, tandis que le commerce des idées est finalement plus restreint et moins vif qu'au temps des cochers, des relais de poste et des presses à bras" (Le Monde, 12 avril 1991, p. 22).

12. Voir Jurt (2006; 2009) e Sapiro (2002).

13. Dans ce contexte il convient de mentionner l'ouvrage Sorá (2003). 
Wittgenstein (PUDAL, 2004) ou Simmel (PINTO, 2009) en France.

D'une manière générale, on s'est rendu compte à l'intérieur de ce groupe de recherche que la réception des biens symboliques dans un autre espace national ou une autre aire linguistique ne peut être mesurée d'une manière normative à l'aune d'une "fidélité" par rapport à un original et pas non plus réduite à une explication vague par l'idée d'une créativité illimitée des récepteurs. Il faudrait plutôt analyser la spécificité des réceptions à travers "une analyse des déterminations concrètes qui s'exercent sur tout acte de lecture" (BOSCHETTI, 2012).

Notamment en ce qui concerne la réception transnationale de la littérature il faudra tenir compte, comme le remarque Gisèle Sapiro, de la politisation des enjeux culturels mais aussi des enjeux économiques qui pèsent sur la production et la circulation des biens symboliques. Mais on devrait également resituer la réception transnationale dans un espace de relations internationales à l'intérieur duquel les relations culturelles sont loin d'être marquées par le principe d'égalité: "La réception d'une littérature dominante sert souvent à modifier les rapports de force constitutifs du champ littéraire d'accueil, celle d'une littérature dominée dans un champ littéraire dominant au niveau international a plus de chance de conforter les représentations indigènes de la culture d'origine et d'être instrumentalisée dans les luttes politiques autour de ces représentations [...]" (SAPIRO, 2006, p. 228).

\section{Références}

BIASI, P-M. De. Vers une science de la littérature. L'analyse des manuscrits et la genèse de l'oeuvre. In: Encyclopedia universalis. Symposium, Paris, 1985, p. 466-476.

BOSCHETTI, A. Le champ littéraire. In: LEBARON, F.; MAUGER. G. (Orgs.). Lectures de Bourdieu. Paris: Ellipses, 2012, p. 243-262.

BOSCHETTI, A. Des revues et des hommes. La Revue des revues, 19, p.51-52, 1994.

BOURDIEU, P. Tout est social. Propos recueillis par Pierre-Marc de Biasi. Magazine littéraire, $n$. $^{\circ}$ 303, p. 104-111, 1992.

BOURDIEU, $P$. Les règles de l'art. Genèse et structure du champ littéraire, Paris: Le Seuil, 1992.

BOURDIEU, P. Le champ littéraire: préalables critiques et principes de méthode. Lendemains, $n^{\circ}$ 36, p. 5-20, 1984.

BOURDIEU, P. Lettre à Paolo Fossati à propos de la Storia dell'arte italiana. Actes de la recherche en sciences sociales, $n^{\circ}$ 31, p. 90-92, 1980.

BOURDIEU, P. Les conditions sociales de la circulation internationale des idées, Romanistische Zeitschrift für Literaturgeschichte/Cahiers d'Histoire des Littératures Romanes, 14, p. 1-10, 1990.

BOURDIEU, P. Quelques propriétés des champs. In: BOURDIEU, P. Questions de sociologie. Paris: Editions de Minuit, 1980, p. 113 -120.

BOUREL, D. Kant en France: un impératif pour tous. In: PINTO, L. (Org.), Le commerce des idées philosophiques. Bellecombe-een-Bauges, Editions du croquant, 2009, p. 19-68.

DIRKX, P. Sociologie et la littérature. Paris: Armand Colin, 2000.

ESPAGNE, M. Le paradigme de l'étranger. Les chaires de littérature étrangère au XIX $\mathrm{XIècle.}^{\mathrm{e}}$ sien Paris: Editions du Cerf, 1993. 
ESPAGNE, M. ; WERNER, M. La construction d'une référence culturelle allemande en France. Genèse et histoire (1750-1914). Annales, 4, p.969992, 1987.

GRÉSILLON, A. Méthodes de lecture. In: HAY, Louis (Org.). Les manuscrits des écrivains. Paris, Hachette -CNRS Éditions, 1993, p. 138-161.

HARRER, D. Französische Literatur in der Weimarer Republik. Zum Verhältnis von Zeitgeschichte und Literaturrezeption bei zeitgenössischen Autoren. Konstanz, HartungGorre Verlag, 1987.

HAY, L. (éd.), Les Manuscrits des écrivains. Paris, Hachette -CNRS Éditions, 1993.

JAUSS, H. R. Literaturgeschichte als Provokation. Francfort/M., Suhrkamp, 1970.

JAUSS, H. R. Ästhetische Erfahrung und literarische Hermeneutik. Francfort/M., Suhrkamp, 1984.

JAUSS, H. R. Pour une herméneutique littéraire. Paris, Gallimard, 1988.

JURT, J. Génétique textuelle et génétique sociale. In: Gifford, P. ; Schmid, M. (Orgs.). La création en acte. Devenir de la critique génétique. Amsterdam/ New York: Rodopi, 2007, p. 41-50.

JURT, J. Génétique sociale. In: BOUJU, E. (Org.). Fragments d'un discours théorique. Nouveaux éléments de lexique littéraire. Rennes: Editions Cécile Defaut, 2015, p. 155-172.

JURT, J. De l'analyse immanente à l'histoire sociale de littérature. A propos des recherches littéraires en Allemagne depuis 1945. Actes de la recherche en sciences sociales, $n^{\circ} 78$, p. 94-101, 1989.

JURT, J. Réceptions littéraires transnationales. L'accueil du naturalisme en Allemagne. In: Charpentier, I. (Org.), Comment sont reçues les œuvres. Actualités de recherches en sociologie de la réception et des publics. Paris: Creaphis, 2006, p. 205-216.
JURT, J. Transnationale Literatur-Rezeption. Am Beispiel der Aufnahme Jean-Luc Benoziglios im deutschsprachigen Raum. Arcadia, t. 44, p.376399, 2009.

JURT, J. Für eine Rezeptionssoziologie. Romanistische Zeitschrift für Literaturgeschichte/ Cahiers d'Histoire des Littératures Romanes, III, p. 214-231, 1979.

JURT, J. La réception de la littérature par la critique journalistique. Paris, 1980.

JURT, J. Les-Arten. Rezeptions- und Lektüreforschung und ihre Folgen für das Literaturverständnis. Revista de Filología Alemana, nº6, p.43-68, 1998.

JURT, J. L'esthétique de la réception - une nouvelle approche de la littérature?. Les Lettres romanes, XXXVII, 3, p. 199-220, 1983.

JURT, J. A descoberta do leitor. Da estética da recepção à sociologia da recepção. In: MELLO, C. M de ; CATHARINA, P. P. G. F.; REIS, S. C. (Orgs.), A palavra, o artista e a leitura - Homenagem a Théophile Gautier. Rio de Janeiro, Confraria do Vento, 2014, p. 29-47.

JURT, J. La réception de la littérature par la critique journalistique. Lectures de Bernanos, 1926-1936. Paris: J.-M. Place, 1980.

JURT, J. Das Konzept der Weltliteratur - ein erster Entwurf eines internationalen literarischen Feldes? In: BACHLEITNER, N.; HALL, M. G. (Org.) Die Bienen fremder Literaturen. Der literarische Transfer zwischen Grossbritannien, Frankreich und dem deutschsprachigen Raum im Zeitalter der Weltliteratur (1770-1850). Wiesbaden: Harrassowitz, 2012, p. 23-44.

JURT, J. Ernst Robert Curtius et le champ universitaire allemand. In: GENTON, F. (Org.), France - Allemagne. De Faust à l'université de masse (Chroniques allemandes 4). Grenoble: CERAAC, 1995, p. 155 -176. 
JURT, J. Vu de l'Allemagne: Les théories littéraires en France. In: PINTO, E. (Org.), Penser l'art et la culture avec les sciences sociales. En l'honneur de Pierre Bourdieu. Paris: Publications de la Sorbonne, 2002, p. 121-137.

JURT, J. De Lanson à teoria do campo literário. Tempo social, v. 16, n 1, p. 29-59, 2004.

LEENHARDT, J. Le 'savoir-lire', ou des modalités socio-historiques de la lecture. Littérature, 70, p. 75-76, 1988.

LEENHARDT, J.; Józsa,P. Lire la lecture. Essai de sociologie de la lecture. Paris: Le Sycomore, 1982, p. 332-341.

MACHEREY, P. Culture nationale et culture cosmopolite chez Mme de Staël. In: ESPAGNE, M.; WERNER M. (Orgs.), Transferts. Les relations culturelles dans l'espace franco-allemand (XVIII ${ }^{e}$ et $\mathrm{XIX}^{\mathrm{e}}$ siècles). Paris: Edition Recherche sur les civilisations, 1988, p. 409-426.

MITTERAND, H. Critique génétique et histoire culturelle. Les dossiers des Rougon-Macquart. In: HAY, L. (Org.), La naissance du texte. Paris: José Corti, 1989, p. 147-162.

NEEFS, J. Critique génétique et histoire littéraire. In: BÉHAR, H.; FAYOLLE, R. (Orgs.). Histoire littéraire aujourd'hui. Paris, Armand Colin, 1990.

PICHÉ, C.Expérience esthétique et herméneutique philosophique. Texte, 3, p. 174-191, 1984.

PINTO, L. Simmel et Tarde. Le retour. In: PINTO, L. (Org.). Le commerce des idées philosophiques. Bellecombe-een-Bauges, Editions du croquant, 2009, p. 137-168.
PUDAL, R. La diffıcile réception de Wittgenstein en France. In : PINTO, L. (Org.). Le commerce des idées philosophiques. Bellecombe-een-Bauges, Editions du croquant, 2009, p. 93-112.

RICOEUR, P. Regards sur l'écriture. In: HAY, L. (Org.), La Naissance du texte. Paris: José Corti, 1989, p. 213-220.

SAPIR0, G. (Org.), Translatio. Le marché de la traduction en France à l'heure de la mondialisation. Paris: CNRS, 2008.

SAPIRO, G. Les contradictions de la globalisation éditoriale. Paris: Nouveau Monde, 2009.

SAPIRO, G. (Org.). L'espace intellectuel en Europe: De la formation des Etats-nation à la mondialisation XIXe - XXIe siècle. Paris, La Découverte, 2009.

SAPIR0, G. Les enjeux politiques de la réception de la littérature hébraïque en France. In: CHARPENTIER, I. (Org.), Comment sont reçues les œuvres. Actualités de recherches en sociologie de la réception et des publics. Paris: Creaphis, 2006, p. 217-228.

SARTRE, J-P. Qu'est-ce que la littérature? Paris, Gallimard, 1948.

SORÁ, G. Traducir el Brasil. Una antropología de la circulación de ideas. Buenos Aires: Libros del Zozal, 2003. 
RESUMO

0 artigo trata da multiplicidade de relações entre literatura e nação. Sobretudo, os casos francófono e germânico são examinados para explorar como a literatura jogou um papel fundador nos processos de constituição das nações modernas, notadamente diante a ausência de estrututuras políticas nacionais. 0 que se distingue de outros contextos nos quais a literatura se tornou um atributo importante de uma nação politicamente constituída.

\section{PALAVRAS-CHAVE}

Literatura. Circulação. Nação.

\section{ABSTRACT}

The article deals with the multiplicity of relationships between literature and nation. Above all, the Francophone and Germanic cases are examined to explore how literature has played a founding role in the constitution processes of modern nations, notably in the absence of national political structures. This differs from other contexts in which literature has become an important attribute of a politically constituted nation.

\section{KEY-WORDS}

Literature. Circulation. Nation. 
